\title{
El rol de Chile en la primera globalización del cobre, $1700-1840$
}

Chile's Role in the First Globalization of Copper, 1700-1840

Juan Navarrete-Montalvo* y Manuel Llorca-Jaña**

\section{RESUMEN}

Este artículo estudia la posición de Chile dentro de las redes mundiales de cobre entre 1700 y la década de 1840. Durante este periodo, los niveles de producción eran bajos, si se los compara con los de 1850-1870 y el siglo XX, debido tanto a las pobres técnicas de extracción y refinado como a los altos costos de transporte. Sin embargo, el cobre chileno fue consumido dentro de Chile, en mercados cercanos como Perú, Bolivia y Argentina, y también se exportó a España, a otros países de Europa, así como a Asia. En esta época, el cobre chileno se había internacionalizado, pero no globalizado. Fue después de la independencia que el cobre chileno comenzó a fluir más libremente a Asia, mientras que los Estados Unidos y Europa continental se convirtieron, también, en mercados claves. Durante este periodo, no solo se exportaba cobre fundido, sino que también regulus y mineral de cobre. Fue solo el arribo de mercantes banqueros británicos a Chile, como Huth \& Co., lo que permitió una verdadera globalización del cobre chileno, tal como muestran los archivos de esta compañía.

\begin{abstract}
This article studies Chile's position within the global copper networks between 1700 and the 1840s. During this period, production levels were low in comparison to the period from 1850-1870 and the 20th century, due to both weak extraction and refinery techniques and high transport costs. However, Chilean copper
\end{abstract}

Palabras clave:

Chile, cobre, globalización, modernidad, Huth.

Keywords:

Chile, copper, globalization, modernity, Huth.

Chileno. Magíster en Historia Económica, Universidad de Santiago de Chile. Investigador Asociado, Universidad de Valparaíso. E-mail: juan.navarrete@uv.cl

Chileno. Doctor en Historia Económica, Leicester University, Reino Unido. Investigador, Escuela de Administración Pública, Universidad de Valparaíso. E-mail: manuel.llorca@uv.cl 
was consumed within Chile, in nearby markets such as Peru, Bolivia and Argentina, and was also exported to Spain and other European countries, as well as Asia. At the time, Chilean copper had gone international, but not yet global. It was after the country's independence that Chilean copper began to flow more freely to Asia, while the United States and continental Europe also became key markets. During this period, the country exported not only molten coppery, but also copper regulus and mineral. The true globalization of Chilean copper only occurred upon the arrival of British banking merchants to Chile, like Huth \& Co., as shown by this company's archives. 
Este artículo pretende situar la posición de Chile dentro de las redes de cobre del mundo antes de la década de 1840, es decir, en el último siglo de dominio colonial español y las primeras décadas desde la independencia de España. Se argumentará que, aun cuando el cobre chileno llegaba a varios mercados internacionales antes de la llegada de mercantes banqueros británicos a Chile después de la Independencia, no fue sino hasta los años de 1820 que el cobre chileno pasó desde ser exportado a unos pocos países a estar verdaderamente globalizado. Esta globalización fue posible únicamente gracias a las redes mundiales que poseían firmas como Huth \& Co.

Los conquistadores españoles llegaron a Chile en 1520, con la expedición de Hernando de Magallanes, que pronto fue seguida por la de Diego de Almagro (1536) y por una tercera, que sería la definitiva, dirigida por Pedro de Valdivia, en 1541. Consiguieron conquistar una parte bastante pequeña del Chile actual, abarcando desde lo que hoy es llamado el Norte Chico (Copiapó, Coquimbo, La Serena) hasta Concepción, en el sur; en esta nueva unidad política (es decir, la Capitanía de Chile, dependiente del Virreinato del Perú) existían ricas vetas de cobre, en particular en el norte y el centro.

Hay evidencias de producción de cobre anterior a estas expediciones de conquista, y también posterior, el cual era consumido localmente, principalmente para la elaboración de utensilios de cocina. En los dos primeros siglos de dominio colonial también hay evidencia no solo de intercambios de barras de cobre y manufacturas brutas de cobre dentro de la Capitanía de Chile, sino también de su distribución dentro de la región, en lo que es hoy Argentina, Bolivia y Perú. En ese momento, la geografía del cobre chileno estaba limitada a las operaciones intrarregionales y los volúmenes exportados eran modestos. Fue solamente durante el periodo cubierto por este artículo que la producción de cobre alcanzó una escala considerable y, con ello, el cobre chileno comenzó a llegar a Europa y Asia, como describiremos con atención más adelante. El arribo de mercantes banqueros británicos a Chile marcó un punto de inflexión: sus amplias redes internacionales permitieron una verdadera globalización del cobre chileno, como bien muestra la evidencia que recabamos en los archivos de Huth \& Co. Esta etapa coincide con el surgimiento del cobre como la primera industria pesada integrada globalmente, con asiento en Gran Bretaña, específicamente en Gales, aunque el rol de Londres en el proceso fue fundamental. 
Este artículo se divide en las siguientes secciones. En primer lugar, tratamos sobre el comienzo de las actividades de cobre en Chile, es decir antes de la llegada de los españoles y hasta el siglo XVII. A continuación, pasamos a centrar nuestra atención en la producción durante el siglo XVIII y las rutas comerciales seguidas durante esa centuria, basados principalmente en fuentes secundarias. Luego se presta especial atención a las exportaciones de cobre chilenas a Asia, Estados Unidos y Europa continental, antes y poco después de la independencia - dado el enfoque de este trabajo, que es la conexión global amplia-, para terminar con las actividades específicas de un mercante banquero bien particular: Huth \& Co. Esta segunda parte descansa en una revisión de fuentes primarias de dicha compañía.

\section{El comienzo de la producción de cobre en Chile}

La producción de cobre en lo que hoy es territorio chileno comenzó mucho antes de la llegada de los españoles, en particular en la Región de Atacama, y era realizada por aimaras, atacameños y diaguitas (Llagostera, 2004; Sutulov y Zauschquevich, 1975; Méndez, 2004). El cobre fue visto como el metal más viable para producir herramientas básicas, debido principalmente a la falta de depósitos de hierro en la región (Sánchez, 2005). Y, en efecto, el cobre se utilizaba para producir varias herramientas, tales como cuchillos, hachas, ollas, jarras, y también para joyería. Más tarde, el cobre refinado chileno comenzó a ser producido también por los conquistadores españoles, desde el siglo XVI, aunque este periodo está escasamente documentado (Cavieres 2003). Los conquistadores comenzaron a exigir cobre para producir cañones y proteger sus fortalezas de los posibles ataques de otras potencias europeas, así como también para elaborar campanas y utensilios de cocina (Pederson, 2008; Sánchez, 2005). En cualquier caso, las principales minas eran las situadas en Carrizal Alto (Región de Atacama), Copiapó, La Serena, Coquimbo, Huasco, Illapel y Aconcagua (Molina 1987 [1810]; Egaña 1803; Aracena, 1884; Dougnac, 1981 y 1991; Fernández, 1894; Cavieres, 2003; Figueroa, 1981). ${ }^{1}$

Según Vicuña Mackenna (1883), el cobre de las zonas del norte era el más adecuado para campanas, mientras que el de la región de Aconcagua era mejor para utensilios de cocina. 
Sin embargo, a pesar de esta conocida producción temprana de cobre refinado, hay consenso en la historiografía respecto de que, durante los dos primeros siglos del periodo colonial, la producción se mantuvo en un nivel extremadamente bajo (ver figura 1), principalmente debido a la escasez de mano de obra (Vicuña Mackenna, 1883; Pederson, 2008; Sánchez, 2005), así como también a los altos costos de transporte por tierra, las técnicas de producción rudimentarias y una demanda restringida. Es igualmente importante considerar que, en cuanto a la actividad minera en general, los principales esfuerzos de la corona española se orientaban hacia la producción de plata y oro. El cobre era aún visto como "la Cenicienta" del sector minero (Figueroa, 1981). En cualquier caso, el cobre refinado en el país estaba destinado principalmente para el consumo doméstico, en particular gracias a la elaboración de ollas y manos de mortero (Vicuña-Mackenna, 1883), así como de campanas y cañones, estos últimos fabricados por fundiciones de artillería en Coquimbo (Molina, 1987 [1810]; Lacoste, 2016). Otro uso importante fue la fabricación de alambiques para producir aguardiente. De acuerdo con Lacoste (2016), las manufacturas de cobre del norte de Chile (alambiques) eran llevadas a otros lugares de Chile o a destinos en el extranjero, tales como Guayaquil (Ecuador), Trujillo, Callao (Perú), Cinti (Bolivia), San Juan², Mendoza, Jujuy y Salta (Argentina).

Además de estas manufacturas de cobre, pequeños excedentes, en forma de barras de cobre refinado, también fueron exportados a Perú (parte de los cuales eran reexportados a otros lugares) y España (Pederson, 2008). De acuerdo con Vicuña Mackenna (1883), la primera exportación de cobre de Chile a Perú tuvo lugar en 1615, para la fundición de la artillería local, debido a un requerimiento de las autoridades de Lima, en consideración de la amenaza latente de una invasión holandesa (véase también Herrmann, 1902 y Pederson, 2008)³. Aquí podemos ver cómo los desarrollos que se iniciaron en Europa (es decir, los Países Bajos) desencadenaron la primera exportación chilena de cobre por vía marítima. En cuanto a España, no es posible establecer una fecha exacta para la primera operación de exportación chilena de

Palomeque (2006) ofrece también pruebas sólidas de la existencia de cobre chileno importado en San Juan y Mendoza, hoy Argentina.

3 El cobre chileno en Perú también era utilizado para producir utensilios de cocina, además de los cañones (Contreras, 2010). 
cobre hacia allí, pero al menos desde 1625 comenzaron a tener lugar algunos envíos regulares (aunque limitados) (Herrmann, 1902).

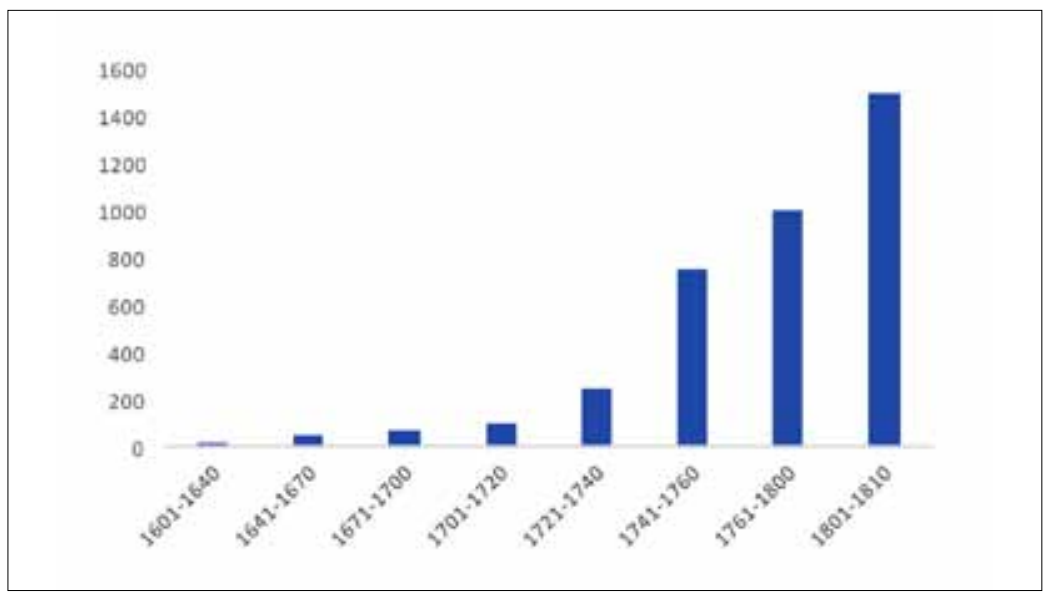

Figura $N^{\circ} 1$. Producción chilena de cobre durante la colonia (medias anuales de toneladas de cobre fino), 1601-1810

Fuente: Herrmann, 1902 y Sululov y Zauschquevich, 1975.

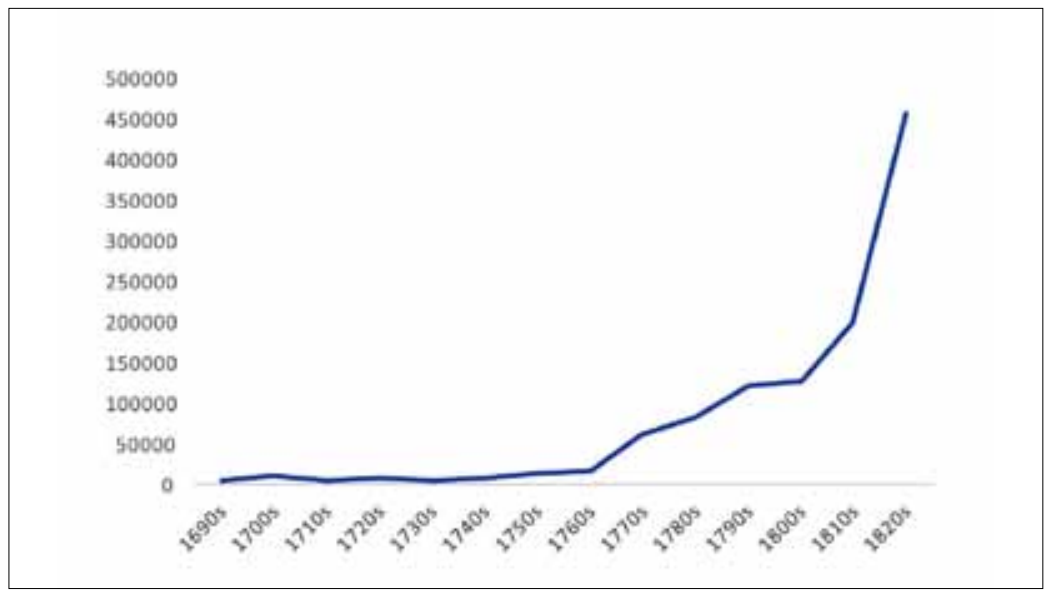

Figura $N^{\circ} 2$. Valor de la producción chilena de cobre durante la época colonial y las primeras décadas de la independencia (en pesos), 1690-1829

Fuente: Carmagnani, 2001.

4 De acuerdo con Méndez (2004), estas cifras deben tomarse con precaución, constituyendo solamente estimaciones aproximadas de la producción real. Y, de hecho, las propias estimaciones de Méndez son más bajas, al menos para la década de 1800, aunque sus cifras no incluyen el contrabando. 
Las técnicas de producción de extracción de minerales de cobre y refinamiento empleadas durante la colonia eran extremadamente rudimentarias (Aracena, 1884; Herrmann, 1902), conllevando altísimos costos de producción. Dicho esto, la producción seguía siendo viable, principalmente a causa de las muy ricas vetas de cobre del país (localizadas en un nivel superficial, fáciles de explotar), lo que permitió una explotación rentable, sin mecanización. Las técnicas de fundición, en particular, eran artesanales y costosas, y utilizaban una gran cantidad de carbón vegetal o leña en los hornos (Fernández, 1894; Molina, 1987 [1810]), lo que llevó a la deforestación en el norte de Chile (Marín, 1920; Herrmann, 1902; Vicuña Mackenna, 1883; Sánchez, 2005). Frecuentemente, el cobre chileno debía ser refinado en el mercado de destino (Sánchez, 2005). En este punto, vale la pena mencionar que los minerales de cobre no solo tenían un contenido muy alto de cobre fino, sino que también se lo hallaba mezclado con oro o plata (Fernández, 1894; Molina, 1987 [1810]), lo que, aunque valioso en sí, planteaba algunos problemas graves para su refinamiento que ralentizaban el proceso (Soler, 2017).

\section{La producción del siglo XVIII y las rutas de exportación}

En lo que se refiere a la parte institucional del sector, la minería en el Chile colonial, incluyendo la producción de cobre, fue regulada por varios cuerpos legales, emitidos en Chile ya desde 1546. Del mismo modo, los códigos de minería coloniales españoles, aplicados por primera vez en México, fueron posteriormente implementados tanto en Perú como en Chile, adaptados a las realidades de ambas entidades políticas (Méndez, 1979). Nueva legislación minera fue introducida en Chile en 1787, basada en un código original, primero promulgado en México y posteriormente modificado en Perú, antes de sufrir el mismo destino en Chile (Méndez, 1979; Dougnac, 1981). De acuerdo con Méndez (1979), esta nueva legislación dio un nuevo impulso a la minería en Chile, gracias a un cambio institucional positivo, mediante la regulación de las condiciones de trabajo y el establecimiento de normas claras para resolver cualquier conflicto potencial. En efecto, un Tribunal de Minería para el reino de Chile se estableció como órgano permanente y específico para la resolución de conflictos sectoriales, así como para velar por el cumplimento de las leyes mineras. De esta 
forma, la nueva codificación y sus instituciones relacionadas trataron de estimular la minería local a través de préstamos a los mineros, establecimiento de gremios y de tribunales, difusión de técnicas de minería, mejoras al suministro de insumos clave para la industria (por ejemplo, herramientas de hierro y pólvora), ${ }^{5} \mathrm{y}$ un registro de todas las minas en el país (Dougnac, 1981; Méndez, 1979; Sánchez, 2005).

Dentro de este marco, durante el siglo XVIII el cobre comenzó a ser producido en niveles superiores, nunca vistos antes, y ahora se exportaba fuera del país en volúmenes mucho mayores, legalmente a Perú ${ }^{6}$ y España - a través de Callao o Buenos Aires (Cruchaga, 1878; Vicuña, Mackenna 1883) - ${ }^{7}$, pero también a menudo a través del contrabando, en el cual eran activos los ingleses, estadounidenses y franceses (Villalobos, 1962; Vicuña Mackenna, 1883; Dougnac, 1999; Pereira, 1936; Sánchez, 2005). ${ }^{8}$ Por lo tanto, el cobre chileno salió de Chile o de Perú, clandestinamente, con dirección a los puertos de Europa y Asia (ver más abajo). Pronto el cobre se convirtió en uno de los principales productos exportados por Chile (Cavieres, 1996) ${ }^{9}$, aunque la producción no era suficiente para hacer frente a la alta demanda en España y en otras regiones (Vicuña Mackenna, 1883). El contrabando del metal

\footnotetext{
$5 \quad$ El alto precio de la pólvora era, por lo general, señalado como otro obstáculo para una producción de cobre más alta (Dougnac, 1981).

$6 \quad$ Las barras y el labrado (regulus) de cobre, junto al trigo, eran, por mucho, los principales productos exportados a Perú desde Chile durante el siglo XVIII. En Perú se lo utilizaba principalmente para grandes platos en la industria azucarera, así como en la producción de cañones y campanas (Molina, 1987 [1810]; Ross, 1894; Sánchez, 2005). Para operaciones comerciales específicas de cobre chileno enviado a Perú y consumido allí o reexportado a Cádiz durante la década de 1770, véase Archivo General de la Nación, Perú, Real Aduana, C. 16.585-54, 1774; C. 16.617-206, Registros de navíos procedentes de los Mares del Sur; Real Aduana, C. 16.590-78, 1774.

7 En España, el cobre chileno era utilizado por las fundiciones de artillería para producir cañones (Molina, 1987 [1810]). Chile era visto por la corona española como una fuente alternativa de cobre, para no depender únicamente de Suecia y Rusia como proveedores (Soler, 2017). Otras colonias americano-españolas que proveían cobre a España eran Cuba y México, pero su oferta no era suficiente para satisfacer la demanda de la corona española. Los excedentes mexicanos eran exportados a Cuba, para abastecer a la industria azucarera en auge (Sánchez, 2005; Cavieres, 2003). En contraste, muy poco cobre se extrae de Perú, donde en 1790, por ejemplo, solo cuatro minas de cobre estaban en funcionamiento. Sobre la producción de cobre marginal del Perú, véase también Contreras (2010).

$8 \quad$ Muchos supuestos balleneros que patrullaban las costas chilenas participaban, en realidad, en el comercio de contrabando, incluyendo el del cobre (Pereira, 1936 y 1971; Villalobos, 1962).

9 Antes, Chile exportaba principalmente oro, plata, trigo y algunos productos ganaderos, tales como cueros, carne salada y sebo (Cavieres, 2003).
} 
rojo va a surgir en respuesta a varias obligaciones impuestas por la corona española sobre el comercio del cobre o sobre su producción, incluyendo un impuesto a la producción de $20 \%$ sobre el cobre valorado en "boca mina"; $3 \%$ de la alcabala, un impuesto sobre la primera venta realizada por los mineros a los comerciantes, a partir de 1778 , que se combinó, en 1787, con otro impuesto para financiar el Tribunal de la Minería (Fernández, 1894; Méndez, 2004; Marín, 1920). Sin embargo, los altos costos de transporte, debidos a la falta de caminos adecuados, los altos costos de producción, la falta de inversiones (y en general de capital), la escasez de pólvora y herramientas de hierro para trabajar las minas, y la debilidad de los derechos de propiedad, impidieron que la producción y las exportaciones alcanzaran niveles más altos (Fernández, 1894; Sánchez, 2005).

En este punto hay que mencionar que el aumento de la producción de cobre en Chile se activó por acontecimientos en la metrópoli. España no tenía una producción propia suficiente de cobre para cubrir su propia demanda ${ }^{10}$, por lo que, a partir de la década de 1570, la corona española firmó tratados comerciales con varios comerciantes para aprovisionarse de cobre con proveedores cercanos, en particular para obtener cobre húngaro y sueco (Soler, 2017). La corona necesitaba cobre para las fundiciones de artillería, tanto en casa (en Ferrol-Galicia, Sevilla y Barcelona, en particular, tenía importantes fundiciones de cobre $)^{11}$ como en las colonias americanas y en la frontera africana. El cobre también se utilizaba en España para la acuñación de monedas vellón ( $50 \%$ de cobre y $50 \%$ de plata) y para la producción de latón (una aleación de cobre y zinc) en Alcaraz-Albacete (Romano, 1965; Cavieres, 2003 y 2003b; Sánchez, 2005).

Cuando el precio del cobre sueco ${ }^{12}$ y húngaro comenzó a aumentar, la corona española buscó fuentes alternativas de suministro, esta vez en el continente americano, siendo Chile uno de los candidatos para el

\footnotetext{
10 Hubo algo de refinación de cobre en España, especialmente en Río Tinto, pero era de mala calidad y los costos de producción eran altos, como en Chile (Cavieres, 2003; Sánchez, 2005). Después de todo, las técnicas chilenas de producción cuprífera eran las heredadas de España.

11 Solamente las artillerías de Barcelona y Sevilla demandaban unas 345 toneladas de cobre fino al año en la década de 1790 (Cavieres, 2003).

12 La famosa mina de cobre Falu vio su producción disminuir desde las primeras décadas del siglo XVIII.
} 
suministro (Sánchez, 2005). Aunque la producción de cobre ya existía en Chile, se organizaron nuevas empresas siguiendo las órdenes de trabajo de la Corona, o aquellas en operación aumentaron sus niveles de producción para cubrir la demanda de España, en particular a partir de 1744, después de la llegada de un real decreto que ordenaba producir tanto cobre como fuera posible (Cavieres, 2003; Carmagnani, 2001). ${ }^{13}$ La corona española comenzó a emitir órdenes regulares para adquirir cobre chileno que debía ser enviado a Lima y, desde allí, a España (Cavieres, 2003). En tiempos de escasez aguda de cobre en la metrópolis, la corona ordenó incluso la incautación de cualquier cobre disponible en Chile, a precios dictados por los oficiales reales, en lugar de precios de mercado, en detrimento de los productores locales (Sánchez, 2005). Por lo tanto, y solo desde este momento en adelante, podríamos decir que el cobre chileno disfrutó de una demanda externa permanente (Carmagnani, 2001) $)^{14}$, aunque bajo condiciones injustas, impuestas por la corona, lo que no constituía en absoluto un estímulo para la producción local.

En cualquier caso, fue el aumento de la demanda de España el que provocó un aumento en la producción de Chile, aunque sin estas condiciones comerciales arbitrarias los niveles de producción podrían haber sido mayores. En otras partes de Europa (fuera de España), la demanda de cobre también aumentó en la segunda mitad del siglo XVIII, debido a su nuevo uso en la construcción de buques, en particular para cubrir los cascos de madera (Sánchez, 2005; Méndez, 2009). Esta demanda tenía que, necesariamente, ser cubierta con el contrabando, ya que España no permitía que sus colonias comerciaran directamente con otras potencias. En cualquier caso, con el tiempo, Chile logró producir alrededor del $10 \%$ del cobre mundial durante 1810-1820, un

\footnotetext{
13 De acuerdo con Sánchez (2005), muchas minas de cobre fueron trabajadas solo en respuesta a órdenes particulares emitidas en España, y fueron abandonadas poco después de que se alcanzó la cuota de producción solicitada. Y, de hecho, se añadieron nuevos centros de producción (por ejemplo, en Copiapó) a los más tradicionales en el área de Coquimbo-La Serena, ampliando así la frontera del cobre en Chile.

14 Tan interesada estaba la corona española en obtener cobre chileno que, desde 1787, a cualquier productor de cobre se le concedía el privilegio de ser un miembro de la nobleza (Dougnac, 1973).
} 
ranking que luego fue liderado por Gran Bretaña y Japón (al menos circa 1800, Sutulov y Zauschquevich, 1975). ${ }^{15}$

En cuanto a las rutas comerciales seguidas por el cobre chileno fuera del país, si se exportaba a través de Buenos Aires, ya legal (necesariamente a España) o ilegalmente, era transportado por tierra desde los centros mineros del norte a Aconcagua (o Valparaíso), y desde allí (por ejemplo, San Felipe y Los Andes) cruzaba la cordillera de Los Andes (en mulas) hasta Mendoza, donde el cobre se cargaba en carretas tiradas por bueyes para ser llevado a Buenos Aires (Méndez, 2009; Cavieres, 2003). Las mulas jugaron un papel crucial en el transporte por tierra del recurso (así como de todos los otros productos, ya fueran minerales o agrícolas) en el Chile colonial. Eran capaces de transportar entre 160 y 180 kilogramos por viaje (Méndez, 2009; Cavieres, 2003). ${ }^{16}$ Desde Buenos Aires el cobre era transbordado a Montevideo y, desde allí, a España (Molina, 1987 [1810]; Soler, 2017; Cavieres, 2003), si se exportaba legalmente, o hacia otros puertos europeos, como Hamburgo, Marsella y Burdeos (Villalobos, 1962). ${ }^{17}$

La alternativa era enviar el cobre a Callao-Lima (ya sea desde Valparaíso, Huasco u otros puertos ${ }^{18}$, donde era transbordado hacia el norte y luego transbordado una vez más, ahora en Panamá (Portobello) o en el lago de Nicaragua, antes de continuar hacia España. ${ }^{19}$ De acuerdo con Carmagnani (2001), el costo final del envío del cobre por tierra (vía Buenos Aires) a Cádiz era bastante similar al que tenían las rutas La Serena-Callao-Cádiz o Valparaíso-Callao-Cádiz, mientras

\footnotetext{
15 El nivel más alto de la participación de Chile en la producción mundial de cobre se alcanzó más adelante. Durante la década de 1860, Chile generaba el 51\% de la producción mundial. Ver Culver y Reinhart (1989).

16 Tan pronto como la minería se convirtió en una actividad económica importante en Chile, la carga más importante realizada por las mulas era la de los minerales, desde las minas hasta los puertos (Méndez, 2009).

17 La ruta a través de Buenos Aires adquirió mayor importancia una vez que el Virreinato del Río de la Plata fue creado en 1776, siendo esta medida parte de las reformas borbónicas (Cavieres, 2003).

18 Si era exportado por vía marítima desde Valparaíso, el cobre producido en el norte - por ejemplo, en Coquimbo - tenía que viajar por tierra de todos modos unos seiscientos o setecientos kilómetros antes de ser puesto a bordo de un buque (Cavieres, 2003).

19 De acuerdo con Cavieres (2003), antes de la década de 1770, en lo que se refiere al cobre, la ruta Callao-Panamá fue la más importante para conectar Chile con España. A su vez, de acuerdo con Vicuña Mackenna (1883), el cobre chileno enviado a Lima fue también a menudo despachado a España a través Guayaquil-Acapulco-La Habana (Cuba). Véase también Mazzeo (2010).
} 
que, según Sánchez (2005), los costos de la ruta a través del Río de la Plata eran inferiores a los de la ruta Callao. Estas rutas comerciales son un buen ejemplo de la integración regional de las colonias hispanoamericanas, como fue destacado hace muchas décadas por Sempat Assadourian (1973 y 1982) y otros (Cavieres, 2003). Siguiendo a Cavieres (2003), el cobre chileno estaba conectado a la economía internacional ya sea a través de Callao, Cádiz o Buenos Aires.

Tan simples como puedan parecer, estas conexiones no eran fáciles de ejecutar. Existían sofisticadas redes comerciales para llevar el cobre fuera del país. De acuerdo con Soler (2017), en el último cuarto del siglo XVIII había más de 35 proveedores de cobre en Chile. Recibían financiación (avances) de ricos comerciantes locales a cambio de la producción futura. Otros actores importantes fueron los transportistas, principalmente peruanos (Cavieres, 1996), que no eran los comerciantes que participaban en la financiación de la producción. Generalmente, todo estaba coordinado por un "agente principal" localizado en, por ejemplo, Cádiz (v. g., la poderosa casa Ustáriz \& Co.), con agentes en Lima y Chile (Cavieres, 2003; Soler, 2017). ${ }^{20}$ Los comerciantes de Lima, en particular, eran intermediarios indeseados en el comercio de exportación de Chile, puesto que dictaban los precios y las condiciones del contrato (Cavieres, 1996; Assadurian, 1984). En otras palabras, Chile tuvo una doble dependencia: de España y de Lima (Romano, 1965; Villalobos, 1962; Carmagnani, 1999; Cavieres, 2003).

Para evitar tantos transbordos y costosos fletes terrestres, el cobre podía ser enviado legalmente a España utilizando principalmente rutas marítimas, a través del Cabo de Hornos o el estrecho de Magallanes, ya sea tocando primero Callao o directamente desde Chile. El Cabo de Hornos (o Estrecho de Magallanes) era una ruta alternativa que comenzó a ser utilizada desde la década de 1740 (Cavieres, 2003; Villalo-

$20 \quad$ Ustáriz \& Co. controlaba cerca de la mitad de todas las exportaciones de cobre de Valparaíso a Callao entre 1761 y 1772, mientras que también eran importantes José de Toro, Nicolás de la Cruz, Ignacio Alcalde y los Cinco Gremios de Madrid. Otros exportadores, no más de veinticinco en total, controlaban la otra mitad. Intermediarios importantes fueron Francisco Javier Errázuriz, Pedro Fermín de Necochea, Antonio de la Lastra, Francisco Astaburuaga, Juan Cranisbro y Salvador Trucios, entre unos pocos más (Cavieres, 2003; Soler, 2017; Méndez, 2009). 
bos 1962$)^{21}$, una vez que estas rutas se abrieron al comercio directo con España con las reformas borbónicas del último cuarto del siglo XVIII (Parry, 1971; Dougnac, 1999; Cavieres, 1996; Fernández, 1894; Molina, 1987 [1810]; Soler, 2017; Parry, 1974). ${ }^{22}$ Los principales puertos de salida eran Caldera (Copiapó), Huasco, Coquimbo y Valparaíso, en menor medida $^{23}$, pero el cobre también tenía que ser transportado desde las minas a los puertos en mulas (como ya se mencionó), de modo que una cierta carga por tierra era inevitable (Cruchaga, 1878; Fernández, 1894; Cavieres, 2003; Méndez, 2004). ${ }^{24}$

Pero estas rutas también abrieron la puerta al aumento del comercio ilegal, en particular después de que los navíos de registro fueran admitidos en los puertos chilenos. Y, en efecto, de acuerdo con Villalobos (1962), a partir de 1740 no era raro ver embarcaciones francesas, británicas y estadounidenses en Chile recogiendo cobre. Por último, en 1797, a causa de la guerra anglo-española (1796-1808), se permitió a los buques "neutrales" comerciar con la América española, en teoría a través de España, pero este sistema alentó en gran medida el contrabando: los comerciantes estadounidenses, británicos (indirectamente, con otra bandera) y alemanes, por ejemplo, no tardaron en comenzar a negociar directamente con muchas colonias americanas españolas, incluyendo Chile y el Río de la Plata. Como parte de estos intercam-

\footnotetext{
${ }_{21}$ En 1740, la ruta de cabo de Hornos fue abierta a navíos de registro (es decir, barcos individuales en lugar de todo el sistema de flotas, a los que se les permitía comerciar directamente entre España y algunos puertos de Hispanoamérica). Antes de eso, a pesar del conocimiento de la existencia de esta ruta, la misma estaba cerrada por decisión española. Chile, por primera vez, podía ahora negociar directamente con España. Este fue un cambio tremendo en las redes comerciales de Chile con el resto del mundo. Y, de hecho, el tráfico a través del Cabo de Hornos aumentó dramáticamente (Villalobos, 1962; Parry, 1971; Carmagnani, 1999; Brading, 1984).

22 Por ejemplo, como parte de estas reformas, el sistema de convoyes terminó y los puertos hispanoamericanos fueron abiertos al comercio directamente con otros puertos españoles, además de Cádiz, mientras que a las colonias se les concedió el derecho a participar en el intercambio comercial directo con otras partes del Imperio español, lo que, en el caso chileno, tuvo lugar a partir de 1778. A todas las colonias también se les permitía comerciar directamente con España (Carmagnani, 1999; Lynch, 1958).

${ }^{23}$ Méndez (2004) fue la primera en restar importancia a Valparaíso, al menos para 1800-1830.

${ }^{24}$ No disponemos de cifras de exportaciones por puertos durante largos periodos de tiempo, pero en la década de 1790 Coquimbo era mucho más importante que Valparaíso, de acuerdo con Vicuña Mackenna (1883).
} 
bios, el cobre fue enviado extensivamente, desde Huasco y Coquimbo, directamente a Europa (Villalobos, 1962). ${ }^{25}$

\section{La conexión con Asia y el comercio de cobre después de la independencia}

Desde principios del siglo XVIII se cree que hubo contactos ilegales directos entre los puertos españoles de América del Sur y Asia. ${ }^{26}$ Por ejemplo, los buques franceses comerciaban, clandestina pero regularmente, entre Callao, pequeños puertos del norte de Chile y China, y así también lo hacían comerciantes estadounidenses, como Cornelius Cole, que dirigía operaciones comerciales entre Coquimbo y Cantón, sobre lo que existe evidencia documental (Pereira, 1971; Villalobos, $1962)^{27}$. Los vínculos entre la América española y Asia aumentaron después de 1785, cuando se creó la Compañía de Filipinas, que promovió el comercio entre España, la América española y Asia (además del realizado por el Galeón de Manila, que se limitó a México), a través del cabo de Hornos o el cabo de Buena Esperanza. Como parte de esto, Pereira (1971) ha dejado excelentes informes de envíos regulares de cobre del norte de Chile a Cantón, realizados por comerciantes estadounidenses en los primeros años del siglo XIX, antes de la independencia, los que proporcionaban beneficios brutos de alrededor del cien por ciento sobre el capital invertido y eran apoyados por una extensa red comercial, en la que participaban las autoridades locales, permitiendo este comercio ilegal. De acuerdo con Pereira, durante el corto periodo 1801-1808, trece mil toneladas de cobre refinado dejaron Coquimbo con dirección a Cantón.

Después de la independencia (1817), mientras el mercado británico se mantuvo "cerrado" para el cobre chileno, dados los elevados derechos de importación y la alta producción de Cornualles, Asia (y también Estados Unidos) se mantuvo durante un periodo como el principal mercado para las exportaciones chilenas de cobre. En cuanto

\footnotetext{
${ }^{25}$ Sobre este periodo y el contrabando británico en la América española, véase Pearce (2014).

26 Más conocido en los intercambios entre Asia y América es Galeón de Manila, legal, que llevó a cabo el comercio directo entre Filipinas y México.

${ }_{27}^{27}$ No solo el cobre chileno fue enviado a China, India y Filipinas, también las pieles de lobo de mar y el aceite de ballena. A cambio, sedas, algodones, té y otros productos asiáticos llegaban a Chile (Pereira, 1948).
} 
a Asia en particular, India se convirtió inmediatamente en el mercado más importante para el cobre chileno (Vicuña, 1883; Kinsbruner, 1972). En 1819, un comerciante local, Agustín Eyzaguirre (junto con otros hombres de negocios), fundó la Compañía de Calcuta, que enviaba principalmente cobre a Asia a cambio de té, sedas, algodones y otros productos (Pereira, 1948). Del mismo modo, China siguió siendo un mercado importante dentro de Asia. Entre 1816 y 1824 llegaron a las costas chilenas "country ships" de Calcuta para recoger un $67 \%$ del cobre chileno. En 1820, un comerciante británico informaba que, de los 21 barcos extranjeros anclados en Valparaíso, cuatro procedían de la India y habían llegado para recoger cobre ${ }^{28}$. De acuerdo con un visitante británico, en Chile, a principios de la década de 1820, "casi todo el cobre producido en el país era exportado en su estado crudo a las Indias Orientales, sus islas y China, a cambio de bienes manufacturados" (Miers, 1826). Precisando estas cifras, se cree que al menos el 75\% de todo el cobre chileno producido entre 1817 y 1825 terminó en puertos asiáticos, en particular en Calcuta (Véliz, 1961).

Del mismo modo, el cobre chileno comenzó a ser enviado a los Estados Unidos también, así como a Europa continental, y en cantidades cada vez mayores (Llorca-Jaña, 2017; Méndez, 2004). Desafortunadamente, no hay datos sólidos para las exportaciones chilenas antes de 1844, lo que permitiría una evaluación más precisa de la importancia relativa de los Estados Unidos. Sin embargo, sabemos que antes de 1835 las exportaciones chilenas de cobre consistían principalmente en barras (Vicuña Mackenna, 1883: 439) y que las exportaciones chilenas a Asia disminuyeron después de $c .1824 .{ }^{29} \mathrm{~A}$ partir de entonces, Estados Unidos se convirtió en el mayor comprador de barras chilenas, en particular durante $c .1834-1843 .{ }^{30}$ Un cónsul británico confirmó esto en 1845: "el metal de cobre se exporta principalmente a través de Valparaí-

\footnotetext{
${ }_{28} \quad$ Biblioteca Guildhall. Documentos de Gibbs. MS 19867. Moens a Gibbs (Londres). Valparaíso, 15-XI-1820.

${ }_{29}$ Sin embargo, no desapareció. Aun en 1840, Alexander Walker informaba que un barco cargado de cobre dejó Huasco para la India. FO 16/43. Huasco, 31-XII-1840. Un año más tarde, Walpole también reportaba que se efectuaban grandes cargamentos de cobre a Asia. FO 16/46. Santiago, 11-V-1842. Del mismo modo, en 1843 Rouse hablaba de cobre chileno despachado a Calcuta. FO 16/51. Valparaíso, 6-III-1843.

${ }_{30}$ FO 132/18. Rouse a Walpole (Santiago). Valparaíso, 28-IV-1840 y Valenzuela, 1992: 535.
} 
so a los Estados Unidos". ${ }^{31}$ De hecho, los primeros datos disponibles en fuentes chilenas apoyan esta afirmación: durante 1844-1849, el 55\% de las barras chilenas fue al país norteamericano (Llorca-Jaña, 2017).

Las décadas siguientes son testigos de una expansión geográfica adicional de las exportaciones chilenas de cobre. No solo se continuó exportando cobre refinado, sino también regulus y mineral de cobre. Durante 1821-1835, la tasa de producción promedio casi duplicó la de la década de 1800-1810, aunque todavía se mantuvo en un nivel bajo. Entre 1836-1843, la producción de cobre nuevamente duplicó la de los 15 años anteriores, alcanzando el equivalente a 6.500 toneladas por año. La fundición de cobre ganó en importancia después de la década de 1830, cuando los hornos de reverbero y otras técnicas fueron ampliamente introducidos. Durante 1840-1860, hubo nuevos aumentos, alcanzándose el valor más alto en la década de 1870, con una producción de más de 45.000 toneladas anuales. Chile estaba produciendo ahora 30 veces más cobre que en la década de $1810^{32} \mathrm{y}$, hacia mediados del siglo XIX, se había convertido en el mayor productor mundial (a expensas de Gran Bretaña), permaneciendo en esa posición durante más de un cuarto de siglo (Przeworski, 1980: 1), antes de ser superado por Estados Unidos durante la década de 1880 (Culver y Reinhart, 1989: 726). No es sorprendente que el periodo comprendido entre $c .1850$ y mediados de la década de 1870 haya sido llamado la "edad de oro" de la industria del cobre chileno (Ortega, 2009: 22; Valenzuela, 1995: 109).

\section{El rol de los mercantes banqueros: Huth \& Co.}

Actores clave en la globalización del cobre chileno fueron los mercantes banqueros británicos que se establecieron en Chile a partir de la Independencia, en particular la casa de Huth \& Co. Es complejo caracterizar acabadamente la figura de los comerciantes-banqueros, debido a que constituyen un grupo heterogéneo, que carece de un perfil único, tanto en medios como en fines; con todo, es posible constatar algunas actividades comunes. Estas incluyen comercialización de bienes a tra-

\footnotetext{
$31 \quad$ FO 133/12. Walpole al conde de Aberdeen (Londres). Santiago, 27-VI-1845.

32 La fundición y refinamiento de cobre chileno fue probablemente la primera "manifestación industrial" de la economía chilena (Pinto, 1994: 62).
} 
vés de sociedades o por cuenta propia, u obtención de consignaciones de productos (por ejemplo, cobre) de manera personal o para terceras personas; comercio de valores; financiamiento del comercio internacional (por ejemplo, de productos primarios como mineral de cobre, pero también de bienes intermedios o manufacturados, tales como barras de cobre); evaluación de riesgos o corretaje; negociación de tasas de comercio internacional u obtención de la aprobación de billetes de intercambio en favor de otros comerciantes; envíos o corretaje de envíos, y emisión de deuda pública y privada (Llorca-Jaña, 2014a).

Antes de 1850, la mayoría de los comerciantes-banqueros de Londres permanecían cautelosos y no diversificaban el área geográfica de sus operaciones. Pero Huth \&. Co. tomó un camino completamente diferente. Estableció una impresionante red global de comercio y préstamos. Casualmente, fue justamente en este periodo cuando la industria del cobre galesa comenzó a volverse un fenómeno verdaderamente global, a través de la obtención de mineral de cobre de muchas partes del mundo y la exportación de cobre refinado a un área geográfica mayor. A su vez, Huth financiaba con entusiasmo parte de este tráfico.

De este modo, en esta sección final tratamos principalmente sobre las actividades de Huth en el cobre chileno, con el objetivo de destacar todas las complejas conexiones construidas por Huth desde Chile en la industria, pero apoyadas por la casa central londinense, gracias a su extensa red global. Por tanto, este artículo ilumina sobre nuestro entendimiento de la economía global inmediatamente posterior a las guerras napoleónicas, incluyendo el surgimiento del cobre como una industria global, y el rol cumplido por las conexiones de Huth en el cobre en este sistema global de comercio y redes; los comerciantesbanqueros londinenses durante 1820-1840, en particular en Chile, un periodo verdaderamente importante en la historia económica chilena, tras la independencia con España (es decir, negocios durante la descolonización); las relaciones financieras anglo-chilenas a principios del siglo XIX, y el comercio exterior chileno en general.

Permítasenos aclarar que, aunque los principales negocios de Huth en cobre durante 1820-1840 estaban relacionados con cobre chileno, la compañía también negociaba con cobre de otros orígenes. Huth transaba con cobre refinado británico y cobre noruego. Si los comerciantes en España (o en cualquier parte) le pedían a Huth cobre refinado britá- 
nico y Huth tenía muchos "amigos" en España ${ }^{33}$, entonces a la compañía no le interesaba la nacionalidad del cobre a obtener; simplemente enviaba el cobre que pudiera obtener en los mejores términos en el mercado británico del momento. ${ }^{34}$

La actividad central de la rama de Huth en Chile era recibir manufacturas británicas a cambio de productos locales. Los textiles en particular eran la columna vertebral del negocio. A cambio de los textiles británicos y algunos otros productos, la plata, el oro y el cobre chilenos eran enviados a Gran Bretaña y a otras partes del mundo, a veces a través de complejos movimientos multilaterales. Con respecto al cobre en particular, ya en 1823 Huth \& Co. estaba mandando regulus de cobre de Caldera (en el norte de Chile) a Swansea (Gales) ${ }^{35}$, a pesar de las altas tasas de importación que había en Gran Bretaña en esa época para el cobre extranjero. También hay evidencia de que Huth Londres obtenía cobre "peruano" (posiblemente chileno) ya desde 1822 para Ledo \& Son, de Ferrol, un producto destinado a la Fábrica de Calderilla de Jubia, en los alrededores de Ferrol (que era entonces donde se fabricaban las monedas de cobre en España). ${ }^{36}$ Finalmente, después de la apertura de la filial en Liverpool de Huth \& Co., en 1839, esta rama comenzó a gestionar más envíos de exportaciones de cobre chileno a Gran Bretaña, mientras que, al mismo tiempo, arbitraba los precios del cobre entre Londres y Liverpool. ${ }^{37}$ Antes de la apertura de la filial en Liverpool, los agentes de Huth allí (Castellain Schaezler \& Co. y John Bibby \& Co.) estaban a cargo de todos los envíos de cobre que arribaban en Mersey. ${ }^{38}$ Es pertinente mencionar aquí que muchos

\footnotetext{
33 Algunos consumidores españoles usuales de cobre que eran provistos vía Huth eran Benito Santos, de La Coruña, y Juan Veyga, de París y La Habana.

34 Por ejemplo, en una ocasión un cliente español (Salvador Rivera e Hijo, de La Coruña) solicitó a Huth cobre. En respuesta a esta demanda, Huth le ofreció tanto cobre chileno como noruego. Hay evidencia también del traslado de cobre, realizado por Huth, desde Gales a Londres. HPEL-40, Huth \& Co. to Huth \& Co. (Liverpool). Londres, 21-X1843.

35 Archivo Nacional de Chile, Documentos Judiciales de Valparaíso. Volumen 321-14. Huth versus Popp. Valparaíso, 23-IV-1823.

36 Huth Papers, UCL Special Collections, Spanish Letters, (HPSL), HPSL-159, Huth \& Co. to Ledo \& Son (Ferrol). Londres, junio de 1822.

37 Huth Papers, UCL Special Collections, English Letters, (HPEL), HPEL-26, Huth \& Co. to Huth, Gruning \& Co. (Valparaiso). Liverpool, 11-V-1839; HPEL-26, Huth \& Co. to Huth \& Co. (London). Liverpool, 20-V-1839.

${ }_{38}$ HPIL, R/16. Castellain Schaezler \& Co. to Huth \& Co. (London). Liverpool, 24-XII1835; Huth-14, Huth \& Co. to Sothern J. Tonge (Liverpool). Londres, 8-XII-1835.
} 
cargamentos de cobre chileno enviados a Gran Bretaña eran después reexportados por Huth hacia otros mercados de la Europa continental, como París, Antwerp y Rotterdam ${ }^{39}$. Esto no es sorprendente, dado que Europa era el principal mercado para Huth durante la primera mitad el siglo XIX.

Adicionalmente, dado que Huth tenía también conexiones sólidas en los Estados Unidos, las barras de cobre chilenas eran enviadas allí a partir de la década de $1820 .^{40}$ Es pertinente mencionar aquí que, junto a los negocios en Chile, Huth había designado dos agentes generales en los Estados Unidos (además de tener acuerdos con cientos de otros hombres de negocios allí). Eran John W. Perit, de Filadelfia (bien conectado en muchas partes del mundo, en particular a través de parientes poderosos), y Goodhue \& Co., comerciantes adinerados de Nueva York. Perit era también uno de los socios de menor categoría de algunos establecimientos poderosos en China y las Filipinas ${ }^{41}$. Pero quizás es más importante el hecho de que Thomas Russell, de Nueva York, haya sido designado por Huth su agente exclusivo en Estados Unidos para las casas sudamericanas ${ }^{42}$. A su vez, con el objetivo de asegurar los servicios de Russell y su compromiso total, este fue hecho socio menor en los establecimientos de la costa oeste del Pacífico ${ }^{43}$. Desafortunadamente, la designación de Russell solo duró unos pocos años, pero, después de su despido, Huth comenzó a trabajar con Grinnell Minurn \& Co., agentes nuevos en Nueva York, que fueron descritos por la sede de Huth en Liverpool como "el mejor apoyo que nuestros amigos [de la costa oeste] tienen en Estados Unidos".44 Aun después de las salidas de Coit y Russell de la sociedad de Valparaíso, comerciantes norteamericanos, tales como Pope \& Aspinwall, de Filadelfia, usualmente enviaban productos estadounidenses a Chile a cambio de cobre despachado directamente a Estados Unidos o a Liverpoo ${ }^{45}$. Esta fue una conexión

\footnotetext{
$39 \quad$ HPEL-32, Huth \& Co. to Huth \& Co. (Liverpool). Londres, 8-XII-1841.

40 Smith College, Northampton, Massachusetts, Hiram Putnam Papers (HCP). Loose papers. Por ejemplo, ver 'Contract between Sewell \& Patrickson and Huth, Coit \& Co. for twelve hundred quintals of cooper'. Valparaíso, 27-XI-1827.

$41 \quad$ HPEL-14, Huth \& Co. to H. H. Stansfeld (Leeds). Londres, 17-VII-1835.

42 HPEL-22, Huth \& Co. to Huth \& Co. (Liverpool). Londres, 10-I-1839. Para mayores detalles, ver HPEL-17, Huth \& Co. to Thomas Russell (New York). Londres, 22-IV-1837.

43 HPEL-21, Huth \& Co. to Thomas Russell (New York). Londres, 13-XII-1838.

44 HPEL-26, Huth \& Co. to Huth \& Co. (London). Liverpool, 15-V-1839.

45 HPEL-31, Huth \& Co. to Pope \& Aspinwall (Philadelphia). Londres, 7 y 9-II-1841.
} 
heredada de Coit. Otras contrapartes en el país norteamericano, como McCrea \& Co., también recibían cobre chileno a través de Huth, Gruning \& Co.

De igual manera, la sede de Huth en Valparaíso era muy activa en la exportación de barras de cobre chilenas al mercado francés para pagar por cargamentos de manufacturas francesas enviadas por Adolphe Roux de París a Chile. ${ }^{46} \mathrm{~A}$ comienzos de la década de 1830, Huth formalizó una sociedad con Roux ${ }^{47}$ con el objetivo de abastecer los establecimientos de la costa oeste con algodones, sedas y otros productos, directos desde Francia o a través de Liverpool/Londres. ${ }^{48} \mathrm{~A}$ su vez, Roux era un consumidor entusiasta de los productos chilenos, que le eran enviados como remesas. El cobre era preferido por Roux y era enviado directamente a Francia, pero también a Gran Bretaña, para ser liquidado allí ${ }^{49}$ El comercio de cobre era visto como una segunda fuente de beneficios para el rentable comercio textil francés.

Pero Roux no era el único comerciante en la Europa continental que aprovisionaba las filiales de Huth en Chile y que recibía cargamentos de cobre a cambio de ello. El caso de Detmering (de Burdeos) es quizás más interesante que el de Roux, porque las manufacturas francesas que eran enviadas desde Burdeos a Valparaíso podían ser pagadas por Huth con cobre chileno enviado directamente a Francia o, inclusive, a India y China, de acuerdo con una propuesta hecha por Detmering mismo. ${ }^{50}$ En efecto, el caso de Detmering ilustra cómo la rama en Chile estaba conectada de manera fácil y rápida con los comerciantes de Europa continental y de Asia, gracias a las muy activas redes globales de Huth. En todos estos lugares, Huth Londres había establecido co-

\footnotetext{
46 HPEL. Volumen 26, Huth \& Co. to Huth \& Co. (London). Liverpool, 5-I-1839.

47 Para los terminus del acuerdo, ver HPEL-11, Huth \& Co. to Adolphe Roux (Valparaiso). Londres, 18-IV-1833; Huth Papers at the Guildhall Library (GLHP), Londres. GLHP, MS 10700-5, 'Agreement between Mr. Adolphe Roux from Paris and Messrs. Frederick Huth \& Co. of London'. Londres, 1-VI-1839. Ver también Cavieres (1999: 179).

48 HPEL-26, Huth \& Co. to Huth \& Co. (London). Liverpool, 2-III-1839. Las manufacturas francesas podían ser enviadas directamente a Chile o indirectamente, desde El Havre a Londres (o Liverpool), y reenviadas desde Gran Bretaña a Chile y Perú. Ver, por ejemplo, GLHP, MS 10700-5, 'Invoice of 115 packages shipped at Havre to address Frederick Huth \& Co. of London to be re-shipped for Callao for account of Roux of Paris'. París, febrero de 1846.

49 Ver, por ejemplo, HPEL-22, Huth \& Co. to Huth \& Co. (Liverpool). Londres, 5-II1839; HPEL-26, Huth \& Co. to Huth \& Co. (London). Liverpool, 5-I-1839 y 15-II-1839.

50 HPEL-26, Huth \& Co. to Huth \& Co. (London). Liverpool, 31-V-1839 y 28-VI-1839.
} 
nexiones sólidas durante 1810-1830, usualmente vía la designación de agentes de confianza en puertos extranjeros clave, tales como los ya mencionados para Estados Unidos.

En China, por ejemplo, Bibbly \& Co. y Russell Sturgis eran conexiones muy sólidas, así como también lo eran Nye Parkin \& Co., Hathaway \& Co. y Kennedy McGregor \& Co. Estas casas de China proveían té y sedas para las filiales de Huth en América del Sur a cambio de cobre o plata. ${ }^{51}$ En definitiva, es interesante tomar nota de que Huth apoyaba con gusto todas estas ramas de comercio que involucraban cobre chileno y que nunca pasaban por puertos británicos. Es pertinente mencionar aquí que muchas de las transacciones con cobre entre Chile y China no eran necesariamente a cuenta de la casa china o de Huth, Gruning \& Co. De hecho, muchas exportaciones de cobre a China eran a cuenta de Estados Unidos, que asumía el riesgo, aunque usualmente recibía crédito de Huth para financiar estos intercambios "norteamericanos" entre Chile y China. ${ }^{52}$ Esta dimensión crediticia nos lleva a la siguiente sección de este artículo.

Huth, al igual que los otros comerciantes-banqueros británicos del periodo, proveía facilidades de crédito que no estaban disponibles en Chile y que eran a menudo necesarias para la producción nacional, y facilitaban en gran parte el involucramiento de Chile en el comercio internacional. De hecho, sin esta forma de crédito, la mayoría de las operaciones de comercio chilenas en el extranjero no habrían podido realizarse y la producción habría sido menor de lo que era. Considerando en particular el crédito ligado al comercio del cobre, la firma entró en el mercado de varias maneras diferentes; inicialmente adelantó dinero a los productores locales (chilenos o extranjeros) a cambio de consignaciones futuras. Por ejemplo, desde la década de 1820, Huth \& Co. firmó numerosos acuerdos relacionados al despacho de cobre refinado en los puertos de Coquimbo, Huasco o Copiapó. Entre los proveedores estaban los famosos Sewell \& Patrickson y Nixon, Ariztia \&

${ }_{51}$ Ver, por ejemplo, HPEL-17, Huth \& Co. to F. S. Hathaway (Canton). Londres, 14-III1837; HPEL-23, Huth \& Co. to F. S. Hathaway (Canton). Londres, 13-IV-1839; HPEL-30, Huth \& Co. to Huth \& Co. (Liverpool). Londres, 29-X-1840; HPEL-45, Huth to Nye Parkin (Canton). Londres, 24-I-1845.

52 HPEL-9, Huth \& Co. to Russell \& Co. (Canton). Londres, 19-IV-1832. 
Co., quienes, a su vez, recibían adelantos de Huth. ${ }^{53}$ Un contrato típico involucraba que Huth adelantara efectivo 30 días después de firmar el contrato, con cerca del $50-55 \%$ del valor acordado del cobre a ser entregado, por ejemplo, en cuatro o cinco meses (después de la firma del contrato); luego otro $25 \%$ un mes después, y el resto tras el envío de todo el cargamento. ${ }^{54}$ De igual modo, más adelante Huth \& Co. hacía avances a la Mexican and South American Company, una de las empresas fundidoras de cobre más grandes de Chile durante este periodo, y que tenía en Huth su principal fuente de crédito en el país. Aquí estamos hablando de envíos considerables (y adelantos asociados con cargamentos de $£ 15,000-£ 18,000) .{ }^{55}$

Un modo completamente diferente de hacer adelantos ligado a las exportaciones de cobre chilenas se relaciona con el acuerdo previamente mencionado entre Roux y Huth para enviar productos franceses a Chile a cambio de cobre. En este caso, Roux recibió adelantos por parte de estos envíos, siempre que la factura y el conocimiento de embarque fueran endosados a Huth \& Co., y que los seguros marítimos fueran confiados a Huth Londres como un medio adicional de proteger el crédito otorgado por Huth. Así, indirectamente, las remesas de cobre usadas para liquidar estos envíos estaban siendo financiadas por Huth desde Londres.

De igual manera, muchas de las exportaciones de Estados Unidos a Chile, pagadas con cobre chileno enviado a Gran Bretaña, también recibían crédito de Huth. Pero, en ocasiones, las contrapartes en Estados Unidos también solicitaban que Huth, Gruning \& Co. enviara remesas en cobre directamente a los Estados Unidos, y no a Gran Bretaña, incluso si había adelantos de Huth Londres involucrados. Tal era el caso de la compañía antes mencionada Pope \& Aspinwall ${ }^{56}$ y también el de McCrea \& Co., esta última convencida de que invertir los ingresos por

\footnotetext{
$53 \quad$ HCP, 'Contract between Sewell \& Patrickson and Huth, Coit \& Co. for twelve hundred quintals of cooper'. Valparaíso, 27-XI-1827. Se acordaba también una tasa por retrasos, en caso de cualquier demora en el envío del cobre a los puertos arriba mencionados. ${ }_{54}$ HCP, 'Contract between Nixon Aristia \& Co. and Huth, Coit \& Co. for 2500 quintals of copper'. Valparaíso, 3-XI-1827, Box 1, folder, 81.

55 HPEL-62, Huth \& Co. to the Mexican \& South American Company. Londres, 16-X1849; HPEL-63, Huth \& Co. to the Mexican \& South American Company. Londres, 30-I1850; HPEL-66, Huth \& Co. to the Mexican \& South American Company. Londres, 5-X1850. Ver también Valenzuela (1992: 524, 542-543).

56 HPEL-31, Huth \& Co. to Huth \& Co. (London). Liverpool, 17-II-1841.
} 
los cargamentos salientes en cobre era "un rendimiento más ventajoso que las barras" ${ }^{17}$ Como en estos casos habría adelantos de Huth Londres (porque el cobre no terminó en Gran Bretaña), la casa de Londres autorizaba a la filial en Chile a mandar cobre directamente a Estados Unidos, pero solamente si el mineral era enviado a Grinnell Minturn \& Co., de Nueva York, o a John Perit, de Filadelfia, quienes lo vendían en suelo norteamericano, enviaban los montos debidos a Huth de Londres, a cuenta de los adelantos ya realizados, y pagaban el resto al comerciante norteamericano, el que también pagaba los costos de agencia de Grinnell Minturn o de Perit por el financiamiento desde Londres de estas operaciones triangulares. ${ }^{58}$

Además, a menudo casas norteamericanas querían comprar cobre en Chile, pero no tenían productos locales para dar a cambio o efectivo para efectuar la compra en Valparaíso. En estos casos, Huth también otorgaría avances a los estadounidenses para comprar cobre, pero, a cambio, Huth Londres pedía que la filial de Valparaíso cobrara una comisión del 2,5\% por la compra de este; el giro de Huth Londres no excedería el $75 \%$ del valor de la factura; todo el cobre comprado en el marco de este acuerdo debía ser enviado directamente a Grinnell Minturn \& Co., y Huth Londres estaba autorizado a cobrar una comisión del $2 \%$ por su aceptación. Finalmente, otra alternativa era que los comerciantes estadounidenses también propusieran que Huth enviara el cobre que estaban comprando a China y lo cambiaran allí por sedas y té que debían ser despachados directamente a Estados Unidos, complicando aún más el comercio multilateral, al involucrar a Chile, Gran Bretaña, Estados Unidos y China ${ }^{59}$. El hecho de que Thomas Russell estuviera bien conectado con Gideon Nye, de Cantón ${ }^{60}$, y de que Perit fuera un socio menor del establecimiento de Russell Sturgis en esa misma ciudad, debe haber ayudado. Una vez más, el potencial ilimitado para el comercio del cobre de Huth, Gruning \& Co. era impensable sin el respaldo y el crédito de un comerciante-banquero global con sede en Londres.

\footnotetext{
57 HPEL-26, Huth \& Co. to Huth \& Co. (London). Liverpool, 1-IV-1839.

58 HPEL-23, Huth \& Co. to H. V. Ward (Baltimore). Londres, 6-IV-1839; HPEL-31, Huth \& Co. to John W. Perit (Philadelphia). Londres, 3-III-1841.

59 HPEL-26, Huth \& Co. to Huth \& Co. (Londres). Liverpool, 30-III-1839.

60 HPEL-21, Huth \& Co. to Thomas Russell (Nueva York). Londres, 18-X-1838.
} 


\section{Conclusiones}

Las tempranas redes de comercio internacional de cobre chileno apoyan la idea de una globalización moderna temprana del intercambio de metales no preciosos, muy anterior a la llamada "primera globalización" (c. 1870-1914). Durante el siglo XVIII y el periodo 1810-1840, las barras de cobre chilenas eran legalmente exportadas a los países vecinos (Perú, Bolivia y Argentina), así como a la metrópoli (España). Del mismo modo, mediante el contrabando, el cobre chileno llegó a muchos otros mercados, esta vez en Asia, Estados Unidos y Europa continental. Los primeros años después de la independencia de España, el continente asiático se constituyó en el principal destino para el cobre chileno, seguido por Estados Unidos. Sin embargo, el papel de Chile dentro de este proceso de globalización moderna en el comercio de cobre no debe ser sobrestimado. Los niveles de la producción cuprífera en Chile permanecieron bastante bajos hasta que las técnicas de refinamiento británicas fueron ampliamente adoptadas, en la década de 1820.

En cualquier caso, hemos mostrado en este artículo que todas las complejas conexiones en la industria del cobre establecidas desde Chile fueron construidas principalmente desde Londres, gracias a la impresionante red global de los mercantes banqueros, como Huth \& Co., y su conocimiento sobre el mineral. Estas incluían apoyo directo a las exportaciones de cobre chileno a Gran Bretaña, así como también a otras partes del mundo. La singular red global de Huth y la extensiva diversificación de los productos con los que trabajaba brindaban confianza e información a un costo menor que el que se podía obtener en Chile por los exportadores de cobre locales o extranjeros, reduciendo así los riesgos de los intercambios. De esta forma, Huth reducía en gran parte los costos de búsqueda, negociación y generales de la transacción en beneficio de quienes comerciaban con el cobre. Huth se especializaba en superar estos obstáculos al comercio en beneficio de sus corresponsales en un mundo de asimetrías de información. La impresionante red global de contactos con los que contaba, daba a la firma al menos un puñado de corresponsales confiables en casi cada puerto importante en el cual había un comerciante que quisiera negociar con cobre chileno. Este hecho era conocido por muchos comerciantes de todo el mundo y usaban las oficinas de 
Huth en Londres y Chile para estos propósitos. Huth enlazaba con éxito los vendedores con los compradores de cobre en un extenso mundo en el que, de otra forma, hubiera faltado contacto directo con comerciantes fuera de Chile, haciendo posibles transacciones de cobre más grandes y más seguras para sus clientes. Huth reducía efectivamente los costos del intercambio, así como también los riesgos inherentes en el comercio internacional chileno debidos a la falta de información y de confianza, y a la existencia de barreras lingüísticas y culturales.

Por su parte, gracias a la literatura general sobre los comerciantes-banqueros ${ }^{61}$, sabíamos que Londres estaba financiando importaciones, exportaciones y reexportaciones británicas, incluyendo importaciones de cobre de muchas partes del mundo. Sin embargo, no estábamos del todo conscientes del importante rol jugado por los comerciantes-banqueros de Londres en el financiamiento del comercio de terceras partes, que nunca tocaba puertos británicos, incluyendo flujos de cobre desde Chile y hacia puertos más allá de los británicos. No era solamente a los exportadores de Chile a los que faltaba capital y debían recurrir al crédito de Huth. Los comerciantes de Estados Unidos, Francia y Asia también se beneficiaban del financiamiento de Huth. Vale la pena enfatizar que la impresionante diversificación del crédito de Huth, para financiar el comercio mundial, fue de la mano con la diversificación geográfica y de productos por la que atravesó Huth, y el cobre no quedó ajeno a esta tendencia. Finalmente, Huth también hizo una contribución valiosa a las exportaciones de cobre chileno (y, por tanto, a las importaciones de otros países, distintos de Gran Bretaña) al proveer servicios de aseguramiento marítimo en Londres, los cuales no estaban disponibles en Chile en ese momento y presentaban un escaso grado de desarrollo en otras partes.

Fueron las singulares redes globales de Huth, su vasto capital, servicios de aseguramiento, sabias estrategias de protección ante el riesgo, conocimiento comercial y recursos, con muchas filiales y agentes de confianza designados en todos los continentes, lo que permitió a

61 Entre los trabajos más importantes se encuentran Chapman $(1984,1992)$ y Jones $(2000,1987)$. 
Huth, Gruning \& Co. de Valparaíso estar totalmente integrada en la economía mundial desde un comienzo, mucho antes de la revolución del transporte y las comunicaciones de la segunda mitad del siglo XIX. Estas eran épocas en las que los costos de las transacciones seguían siendo altos para cualquier casa comercial en Chile que quisiera realizar intercambios con todo el mundo. El comercio chileno de cobre sin duda se benefició en gran medida de tener a Huth \& Co. como un jugador activo en este mercado en particular. En un plano más amplio, hay pocas dudas sobre el hecho de que firmas como Huth \& Co. contribuyeron extensivamente a la emergencia del cobre como la primera industria pesada globalmente integrada.

\section{Referencias bibliográficas}

Fuentes primarias

a) Fondos Documentales

Archivo Nacional de Chile, Documentos Judiciales de Valparaíso.

Biblioteca Guildhall. Documentos de Gibbs.

Huth Papers, UCL Special Collections, Spanish Letters, (HPSL), University of London.

Huth Papers, UCL Special Collections, English Letters, (HPEL), University of London.

Huth Papers at the Guildhall Library, Londres (GLHP).

Hiram Putnam Papers (HCP), Smith College, Northampton, Massachusetts.

\section{Fuentes secundarias}

a) Artículos y capítulos de libros

Brading, D. A. (1984). "La España de los Borbones y su imperio americano", en L. Bethell (editor). Historia de Cambridge de América Latina, volumen II. Cambridge: Cambridge University Pres, pp. 85-126.

Culver, W. y C. Reinhart (1989). "Sueños capitalistas: la respuesta de Chile a la competencia mundial de cobre del siglo XIX", en Estudios comparativos de sociedad y la historia, Vol. 31, $\mathrm{N}^{\circ} 4$, pp. 722-744. 
Dougnac Rodríguez, A. (1999). "Proyección de las Ordenanzas de Minería de Nueva España en Chile (1787-1874)", en Estudios Histórico-Jurídicos, $\mathrm{N}^{\circ}$ 21, pp.111-158.

Dougnac Rodríguez, A. (1981). "La Real Administración del Importante Cuerpo de Minería de Chile (1787-1802)", en Revista Chilena de Historia del Derecho, $\mathrm{N}^{\circ}$ 8, pp. 109-130.

Dougnac Rodríguez, A. (1973). "Mineros y Asientos de minas en Chile (1787-1817)", en Estudios Históricos, № 18, pp. 49-114.

Figueroa, M. (1981). "Bancos de Fomento Minero en Chile Durante el siglo XVIII", en Revista Chilena de Historia y Geografía, $\mathrm{N}^{\circ}$ 149, pp. 43-66.

Kinsbruner, J. (1972). "La condición política de los residentes comerciantes británicos en Chile durante el gobierno de O'Higgins, 1817-1823", en Las Américas, Vol. 27, pp. 26-39.

Lacoste, P. (2016). "Labrado Cobre, alambique y aguardiente. Chile y Argentina, 1586-1850", en Quinto Sol, Vol. 20, № 1, pp. 1-29.

Llorca-Jaña, M. (2014a). “Shaping Globalization: London's Merchant Bankers in the Early Nineteenth Century", en Business History Review, Vol. 88, ํㅜ 3, pp. 469-495.

Pereira, E. (1948). "Las primeras relaciones comerciales entre Chile y el Oriente", en Boletín de la Academia Chilena de la Historia, Vol. 39, pp. 1-19.

Pereira, E. (1936). "Buques norteamericanos en Chile una multa de la época colonial, 1788-1810", en Anales de la Universidad de Chile, Vol. 21, pp. 90-129.

Sánchez, J. (2005). “El Cobre en Chile en su Etapa chilena (1550-1870)”, en J. A. Uribe (ed.). La Industria del Cobre en la América española. México, Chile, Perú y Cuba, Siglos XVI-XIX. Morelia: UMSNH, pp. 79-132.

Sempat Assadourian, C. (1973). "Modos de producción, capitalismo y subdesarrollo en América Latina”, en AA. VV. Modos de producción en América Latina. Córdoba: Pasado y Presente, pp. 47-77.

Soler, L. (2017). “Tráfico de cobres de los minerales chilenos a Cádiz. Redes transregionales de negocios (1750-1800)", en Tempus, No 5, pp. 25-60.

Valenzuela, L. (1992). "The Chilean copper smelting industry in the mid-nineteenth century: phases of expansion and stagna- 
tion, 1834-58", en Journal of Latin American Studies, Vol. 24, $\mathrm{N}^{\circ}$ 3, pp. 507-550.

Villalobos, S. (1962). "El comercio extranjero a fines de la dominación española", en Revista de Estudios Interamericanos, Vol. 4, N ${ }^{\circ}$ 4, pp. 529-543.

b) Libros

Aracena, F. (1884). La Industria del Cobre en Las Provincias de Atacama y Coquimbo. Valparaíso: Imprenta del Nuevo Mercurio.

Carmagnani, M. (2001). Los mecanismos de la vida económica en una sociedad colonial. Chile 1680-1830. Santiago de Chile: Dirección de Bibliotecas, Archivos y Museos, Centro de Investigaciones Diego Barros Arana.

Cavieres, E. (1996). El comercio chileno en la economía mundo colonial. Valparaíso: PUCV.

Cavieres, E. (1999). Comercio Chileno y Comerciantes Ingleses. Santiago de Chile: Editorial Universitaria.

Cavieres, E. (2003). Servir al soberano sin detrimento del vasallo. Valparaíso: Ediciones Universitarias de la Universidad Católica de Valparaíso.

Chapman, S. D. (1984). The rise of merchant banking. London: George Allen \& Unwin.

Chapman, S. D. (1992). Merchant enterprise in Britain. Cambridge: Cambridge University Press.

Cruchaga, M. (1878). Estudio sobre la organización económica i la hacienda pública de Chile. Santiago de Chile: Imprenta de "Los Tiempos".

Fernández, G. (1894). Minería y Metalurgia colonial en el Reyno de Chile. Una visión A través del Informe de Don Juan Egaña al verdadero Tribunal de Minería de 1803. Santiago de Chile: s / d.

Herrmann, A. (1902). La Producción en Chile de los metales i minerales mas importantes de las ventas naturales, del Azufre i del guano, desde La Conquista hasta multas del año 1902. Santiago de Chile: Barcelona.

Jones, C. (1987). International business in the nineteenth century. Brighton: Wheatsheaf Books.

Jones, G. (2000). Merchants to multinationals. Oxford: Oxford University Press. 
Lynch, J. (1958). Spanish Colonial Administration, 1782-1810: The Intendant System in the Viceroyalty of the Rio de la Plata. Londres: University of London, Athlone Press.

Marín, S. (1920). La Industria del Cobre en Chile. Santiago de Chile: Editorial Universitaria.

Méndez, L. M. (1979). Instituciones y problemas de la minería en Chile: 1787-1826. Santiago de Chile: Editorial Universitaria.

Méndez, L. M. (2009). El comercio minero terrestre entre Chile y Argentina 1800-1840. Caminos, arriería y exportación minera. Santiago de Chile: Universidad de Chile.

Méndez, L. M. (2004). La exportación minera en Chile 1800-1840. Un estudio de historia económica y social en la transición de la Colonia a la República. Santiago de Chile: Editorial Universitaria.

Miers, J. (1826). Travels in Chile and La Plata: Including Accounts Respecting the Geography, Geology, Statistics, Government, Finances, Agriculture, Manners, and Customs, and the Mining Operations in Chile. Dos volúmenes. London: Baldwin, Cradock, y Joy.

Molina, J. I. (1987). Ensayo sobre la historia natural de Chile. Santiago de Chile: Ediciones Maule.

Parry, J. H. (1971). El Imperio Seaborne español. Londres: Hutchinson \& Co.

Parry, J. H. (1974). Comercio y dominio. Los imperios europeos de ultramar en el siglo XVIII. Londres: Cardenal.

Pearce, A. (2014). Comercio británico con la América española, 17631808. Liverpool: Liverpool University Press.

Pederson, L. (2008). La industria minera del Norte Chico. Santiago de Chile: RIL.

Przeworski, J. F. (1980). The decline of the copper industry in Chile and the entrance of North American capital, 1870-1916. Nueva York: Multinational Corporations.

Romano, R. (1965). Una economía colonial: Chile en el siglo XVIII. Buenos Aires: Eudeba.

Ross, A. (1894). Reseña histórica del comercio de Chile durante colonial la época. Santiago de Chile: Cervantes.

Sempat Assadourian, C. (1982). El Sistema de la economía colonial: mercado interno, regiones y espacio económico. Lima: IEP. 
Sutulov, A. y A. Zauschquevich (1975). El cobre chileno. Santiago de Chile: Editorial Universitaria.

Vicuña Mackenna, B. (1883). El libro del cobre i del carbón de piedra en Chile. Santiago de Chile: Cervantes. 